\title{
GENERALIZED BAER RINGS
}

\author{
TAI KEUN KWAK
}

Received 8 May 2006; Revised 6 July 2006; Accepted 18 July 2006

We investigate the question whether the p.q.-Baer center of a ring $R$ can be extended to $R$. We give several counterexamples to this question and consider some conditions under which the answer may be affirmative. The concept of a generalized p.q.-Baer property which is a generalization of Baer property of a ring is also introduced.

Copyright (c) 2006 Hindawi Publishing Corporation. All rights reserved.

\section{Introduction}

In [15], Kaplansky introduced Baer rings as rings in which every right (left) annihilator ideal is generated by an idempotent. According to Clark [9], a ring $R$ is called quasi-Baer if the right annihilator of every right ideal is generated (as a right ideal) by an idempotent. Further works on quasi-Baer rings appear in $[4,6,17]$. Recently, Birkenmeier et al. [8] called a ring $R$ to be a right (resp., left) principally quasi-Baer (or simply right (resp., left) p.q.-Baer) ring if the right (resp., left) annihilator of a principal right (resp., left) ideal is generated by an idempotent. $R$ is called a p.q.-Baer ring if it is both right and left p.q.Baer. The class of right or left p.q.-Baer rings is a nontrivial generalization of the class of quasi-Baer rings. For example, if $R$ is a commutative von Neumann regular ring which is not complete, then $R$ is p.q.-Baer but not quasi-Baer. Observe that every biregular ring is also a p.q.-Baer ring.

A ring satisfying a generalization of Rickart's condition (i.e., every right annihilator of any element is generated (as a right ideal) by an idempotent) has a homological characterization as a right $P P$-ring which is also another generalization of a Baer ring. A ring $R$ is called a right (resp., left) PP-ring if every principal right (resp., left) ideal is projective (equivalently, if the right (resp., left) annihilator of an element of $R$ is generated (as a right (resp., left) ideal) by an idempotent of $R$ ). $R$ is called a $P P$-ring (also called a Rickart ring [3, page 18]) if it is both right and left $P P$. Baer rings are clearly right (left) $P P$-rings, and von Neumann regular rings are also right (left) $P P$-rings by Goodearl [10, Theorem 1.1]. Note that the conditions right $P P$ and right p.q.-Baer are distinct 
[8, Examples 1.3 and 1.5], but $R$ is an abelian PP-ring if and only if $R$ is a reduced p.q.Baer ring [8, Corollary 1.15].

Throughout this paper, $R$ denotes an associative ring with identity. For a nonempty subset $X$ of $R$, we write $r_{R}(X)=\{a \in R \mid X a=0\}$ and $\ell_{R}(X)=\{a \in R \mid a X=0\}$, which are called the right and left annihilators of $X$ in $R$, respectively.

\section{Principally quasi-Baer centers}

As a motivation for this section, we recall the following results.

(1) The center of a Baer ring is Baer [15, Theorem 7].

(2) The center of a quasi-Baer ring is quasi-Baer [7, Proposition 1.8].

(3) The center of a right p.q.-Baer ring is $P P$ (hence p.q.-Baer) [8, Proposition 1.12].

(4) Every reduced PI-ring with the Baer center is a Baer ring [1, Theorem D].

It is natural to ask if the p.q.-Baer center of a ring $R$ can be extended to $R$. In this section, we show that this question has a negative answer, and so we investigate the class of rings with some conditions under which the answer to this question is affirmative.

Let $C(R)$ denote the center of a ring $R$.

Example 2.1. (1) Let $K$ be a field. We consider the $\operatorname{ring} R=K[X, Y, Z]$ with $X Y=X Z=$ $Z X=Y X=0$ and $Y Z \neq Z Y$. Then $R$ is reduced and $C(R)=K[X]$ is Baer and so p.q-Baer. But $r_{R}(Y)$ has no idempotents. Thus $R$ is not right p.q.-Baer. Note that

$$
I=\{f(Y, Z) \in K[Y, Z] \mid f(0,0)=0\}
$$

is a two-sided ideal of $R$ and $I \cap C(R)=0$.

(2) Let

$$
R=\left\{\left(\begin{array}{ccc}
x & y & z \\
0 & x & u \\
0 & 0 & v
\end{array}\right) \mid x, y, z, u, v \in \mathbb{R}\right\} \subseteq \operatorname{Mat}_{3}(\mathbb{R})
$$

where $\mathbb{R}$ denotes the set of real numbers. Then $R$ is a PI-ring which is not semiprime. Then we see that

$$
r_{R}\left(\left(\begin{array}{lll}
0 & 1 & 0 \\
0 & 0 & 0 \\
0 & 0 & 0
\end{array}\right) R\right)=\left\{\left(\begin{array}{lll}
0 & b & c \\
0 & 0 & 0 \\
0 & 0 & 0
\end{array}\right) \mid b, c \in \mathbb{R}\right\}
$$

But this cannot be generated by an idempotent. Hence $R$ is not right p.q.-Baer. On the other hand,

$$
C(R)=\left\{\left(\begin{array}{lll}
x & 0 & 0 \\
0 & x & 0 \\
0 & 0 & x
\end{array}\right) \mid x \in \mathbb{R}\right\} \cong \mathbb{R}
$$

Therefore $C(R)$ is Baer.

Observe that Example 2.1(2) also shows that there exists a PI-ring $R$ with the Baer center, but $R$ is not right p.q.-Baer. 
However, we have the following results.

Lemma 2.2 [8, Proposition 1.7]. $R$ is a right p.q.-Baer ring if and only if the right annihilator of any finitely generated right ideal is generated (as a right ideal) by an idempotent.

Proposition 2.3. Let $R$ be a ring with the p.q.-Baer center $C(R)$. If $R$ satisfies any of the following conditions for any nonzero two-sided ideal I of $R$, then $R$ is quasi-Baer (and hence right p.q.-Baer):

(1) $I \cap C(R)$ is a nonzero finitely generated right ideal of $C(R)$;

(2) $I \cap C(R) \neq 0$ and every central idempotent of $R$ is orthogonal;

(3) $I \cap C(R) \neq 0$ and every right ideal of $R$ generated by a central element contains $C(R)$.

Proof. Let $I$ be a nonzero two-sided ideal of $R$. If $r_{R}(I)=0$, then we are done. Thus we assume that $r_{R}(I) \neq 0$.

(1) By hypothesis and Lemma 2.2, $I \cap C(R) \neq 0$ and $r_{C(R)}(I \cap C(R))=e C(R)$ for some $e^{2}=e \in C(R)$. We claim that $r_{R}(I)=e R$. If $I e \neq 0$, then $I e$ is a nonzero two-sided ideal of $R$. Thus, by hypothesis, $0 \neq I e \cap C(R) \subseteq I \cap C(R)$. Let $0 \neq x \in I e \cap C(R)$. Then $x=$ $y e \in I \cap C(R)$ for some $y \in I$, and so $x=x e=0$; which is a contradiction. Hence $e R \subseteq$ $r_{R}(I)$, and then $r_{R}(I)=R \cap r_{R}(I)=(e R \oplus(1-e) R) \cap r_{R}(I)=e R \oplus\left((1-e) R \cap r_{R}(I)\right)$. We show that $(1-e) R \cap r_{R}(I)=0$. Suppose that $0 \neq(1-e) R \cap r_{R}(I)$. Then $(1-e) R \cap C(R)$ is a nonzero two-sided ideal of $R$. Thus, by hypothesis, $0 \neq(1-e) R \cap r_{R}(I) \cap C(R)=$ $(1-e) R \cap r_{C(R)}(I) \subseteq(1-e) R \cap r_{C(R)}(I \cap C(R)) \subseteq(1-e) R \cap e C(R) \subseteq(1-e) R \cap e R=0 ;$ which is also a contradiction. Therefore $r_{R}(I)=e R$, and thus $R$ is quasi-Baer.

(2) There exists $0 \neq a \in C(R)$ such that $a \in I$, and so $r_{C(R)}(a C(R))=e C(R)$ for some $e^{2}=e \in C(R)$ by hypothesis. Then $r_{R}(a R)=e R$. Since $r_{R}(a R) \cap C(R)=r_{C(R)}(a C(R))=$ $e C(R), e \in r_{R}(a R)$, and so $e R \subseteq r_{R}(a R)$, and thus $r_{R}(a R)=e R$ by the similar method to (1). Hence $r_{R}(I) \subseteq e R$. Now, we claim that $e R \subseteq r_{R}(I)$. If not, there exists $0 \neq x \in R$ such that $x \in I \cap C(R)$ by the same arguments as in $(1)$. Then $r_{C(R)}(x C(R))=f C(R)$ for some $f^{2}=f \in C(R)$, and so $r_{R}(x R)=f R$. Hence $r_{R}(I) \subseteq f R \cap e R=0$; which is a contradiction. Thus $r_{R}(I)=e R$ for some $e^{2}=e \in R$, and therefore $R$ is a quasi-Baer ring.

(3) By hypothesis, there exists $0 \neq a \in I \cap C(R)$, and so $r_{C(R)}(a C(R))=e C(R)$ for some $e^{2}=e \in C(R)$. Then $r_{R}(a R)=e R$, and this implies that $r_{R}(I) \subseteq e R$ by the same method as in (2). Now, we claim that $e R \subseteq r_{R}(I)$. If not, there exists $0 \neq x \in I e \cap C(R) \subseteq I \cap a R \subseteq$ $a R$, by hypothesis. We put $x=y e \in C(R)$ for some $y \in I$. Since $r_{R}(x) \supseteq r_{R}(a R)=e R$, we obtain $x=x e=0$; which is a contradiction. Thus $e R \subseteq r_{R}(I)$, and consequently $r_{R}(I)=$ $e R$. Therefore $R$ is a quasi-Baer ring.

COROLlaRY 2.4. Let $R$ be a semiprime PI-ring with the p.q.-Baer center $C(R)$. If either every central idempotent of $R$ is orthogonal or every right ideal of $R$ generated by a central element contains $C(R)$, then $R$ is quasi-Baer.

The proof follows from [18, Theorem 6.1.28] and Proposition 2.3.

Part (1) of the following example shows that the condition " $I \cap C(R)$ is a nonzero finitely generated right ideal of $C(R)$ " and the condition "every central idempotent of $R$ is orthogonal" in Proposition 2.3(1) and (2) are not superfluous, respectively, and parts (2) and (3) show that in Proposition 2.3, the condition (1) is not equivalent to the condition (2). 
Example 2.5. (1) Let $R=\left\{\left\langle a_{i}\right\rangle \in \prod_{i=1}^{\infty} T_{i} \mid a_{i}\right.$ is eventually constant $\}$, where $T_{i}=\operatorname{Mat}_{2}(F)$ for all $i$ and $F$ is a field. For a two-sided ideal $I=\left\{\left\langle a_{i}\right\rangle \in R \mid a_{i}=0\right.$ if $i$ is even $\}, r_{R}(I)=$ $\left\{\left\langle b_{j}\right\rangle \in R \mid b_{j}=0\right.$ if $i$ is odd $\}$. Since

$$
\left\langle\left(\begin{array}{ll}
1 & 0 \\
0 & 1
\end{array}\right),\left(\begin{array}{ll}
0 & 0 \\
0 & 0
\end{array}\right),\left(\begin{array}{ll}
1 & 0 \\
0 & 1
\end{array}\right),\left(\begin{array}{ll}
0 & 0 \\
0 & 0
\end{array}\right), \ldots\right\rangle \notin R
$$

$r_{R}(I)$ cannot be generated by an idempotent of $R$. Thus $R$ is not quasi-Baer. Note that

$$
C(R)=\left\{\left\langle a_{i}\right\rangle \in R \mid a_{i}=\left(\begin{array}{ll}
k & 0 \\
0 & k
\end{array}\right) \text { for some } k \in F\right\}
$$

is p.q.-Baer. Now,

$$
I \cap C(R)=\left\{\left\langle a_{i}\right\rangle \in R \mid a_{i}=0 \text { if } i \text { is even, } a_{i}=\left(\begin{array}{ll}
x & 0 \\
0 & x
\end{array}\right) \in \operatorname{Mat}_{2}(F) \text { if } i \text { is odd }\right\}
$$

is not finitely generated. Moreover,

$$
\left\langle\left(\begin{array}{ll}
1 & 0 \\
0 & 1
\end{array}\right),\left(\begin{array}{ll}
0 & 0 \\
0 & 0
\end{array}\right),\left(\begin{array}{ll}
0 & 0 \\
0 & 0
\end{array}\right), \ldots\right\rangle, \quad\left\langle\left(\begin{array}{ll}
1 & 0 \\
0 & 1
\end{array}\right),\left(\begin{array}{ll}
1 & 0 \\
0 & 1
\end{array}\right),\left(\begin{array}{ll}
0 & 0 \\
0 & 0
\end{array}\right),\left(\begin{array}{ll}
0 & 0 \\
0 & 0
\end{array}\right), \ldots\right\rangle
$$

are idempotents, but they are not orthogonal.

(2) Let $R=F\left[x_{1}, x_{2}, \ldots\right]$, where $F$ is a field. Then $R$ is a commutative quasi-Baer ring whose only idempotents 0 and 1 are orthogonal, but the two-sided ideal $\left\langle x_{1}^{2}, x_{2}^{2}, \ldots\right\rangle$ of $R$ is not finitely generated.

(3) Let $R=\mathbb{Z} \oplus \mathbb{Z}$. Then $R$ is a commutative quasi-Baer ring. Since $R$ is Noetherian, every two-sided ideal of $R$ is finitely generated. But the central idempotents $(1,0)$ and $(1,1)$ are not orthogonal.

Related to the result of $[1$, Theorem D], we have the next example.

Example 2.6. (1) Let $R=\mathscr{C}[0,1]$ be the ring of all real-valued continuous functions on $[0,1]$. Then $R$ is commutative (and so PI) and reduced. But $R$ is not p.q.-Baer. Let

$$
f:[0,1] \longrightarrow \mathbb{R}
$$

be defined by

$$
f(x)= \begin{cases}0, & 0 \leq x \leq \frac{1}{2} \\ x-\frac{1}{2}, & \frac{1}{2}<x \leq 1\end{cases}
$$


Then $f \in R$, and so

$$
r_{R}(f)=\left\{g \in R \mid g\left(\left(\frac{1}{2}, 1\right]\right)=0\right\} \neq 0
$$

Suppose that $r_{R}(f)=e R$ for some nonzero idempotent $e \in R$. Then $e(x)^{2}=e(x)$, for each $x \in[0,1]$. Thus $e(x)=0$ or $e(x)=1$. Since $e \in r_{R}(f), e((1 / 2,1])=\{0\}$. But $e$ is continuous, and so $e(x)=0$ for each $x \in[0,1]$. Hence $r_{R}(f)=0$; which is a contradiction. Thus $R$ is a reduced PI-ring which is not right p.q.-Baer.

(2) We take the ring in $[12$, Example $2(1)]$. Let $\mathbb{Z}$ be the ring of integers and $\operatorname{Mat}_{2}(\mathbb{Z})$ the $2 \times 2$ full matrix ring over $\mathbb{Z}$. Let

$$
R=\left\{\left(\begin{array}{ll}
a & b \\
c & d
\end{array}\right) \in \operatorname{Mat}_{2}(\mathbb{Z}) \mid a-d \equiv b \equiv c \equiv 0(\bmod 2)\right\} .
$$

Then $R$ is right p.q.-Baer, but $R$ is neither right $P P$ nor left $P P$ by [12, Example 2(1)]. Moreover, it can be easily checked that $R$ is an abelian PI-ring with the $P P$ center.

\section{Generalized p.q.-Baer rings}

Regarding a generalization of Baer rings as well as a $P P$-ring, recall that a ring $R$ is called a generalized right $P P$-ring if for any $x \in R$, the right ideal $x^{n} R$ is projective for some positive integer $n$, depending on $n$, equivalently, if for any $x \in R$, the right annihilator of $x^{n}$ is generated by an idempotent for some positive integer $n$, depending on $n$. Left cases may be defined analogously. A ring $R$ is called a generalized $P P$-ring if it is both generalized right and left $P P$-ring. Right $P P$-rings are generalized right $P P$ obviously. A number of papers have been written on generalized $P P$-rings. For basic and other results on generalized $P P$-rings, see, for example, $[11,14,16]$.

As a parallel definition to the generalized $P P$-property related to the p.q.-Baer property, we define the following.

Definition 3.1. A ring $R$ is called a generalized right p.q.-Baer ring if for any $x \in R$, the right annihilator of $x^{n} R$ is generated by an idempotent for some positive integer $n$, depending on $n$. Left cases is defined analogously. A ring $R$ is called a generalized p.q.-Baer ring if it is both generalized right and left p.q.-Baer ring.

We have the following connections.

Lemma 3.2 [12, Lemma 1]. Let $R$ be a reduced ring. The following are equivalent:

(1) $R$ is right $P P$;

(2) $R$ is $P P$;

(3) $R$ is generalized right $P P$;

(4) $R$ is generalized $P P$;

(5) $R$ is right p.q.-Baer; 
(6) $R$ is p.q.-Baer;

(7) $R$ is generalized right p.q.-Baer;

(8) $R$ is generalized p.q.-Baer;

Shin [19] defined that a ring $R$ satisfies (SI) if for each $a \in R, r_{R}(a)$ is a two-sided ideal of $R$, and proved that $R$ satisfies (SI) if and only if $a b=0$ implies that $a R b=0$ for $a, b \in R$ [19, Lemma 1.2]. The (SI) property was studied in the context of near-rings by Bell, in [2], where it is called the insertion of factors principle (IFP). It is well known that every reduced ring has the IFP, and if $R$ has the IFP then it is abelian, but the converses do not hold, respectively.

Recall from [8, Corollary 1.15] that $R$ is an abelian PP-ring if and only if $R$ is a reduced p.q.-Baer ring. Similarly, we have the following.

Proposition 3.3. Let a ring $R$ have the IFP. Then $R$ is a generalized right PP-ring if and only if $R$ is a generalized right p.q.-Baer ring.

Proof. For any $x \in R$ and positive integer $n, r_{R}\left(x^{n}\right)=r_{R}\left(x^{n} R\right)$ since $R$ has the IFP.

Every right p.q.-Baer rings is a generalized right p.q.-Baer, but the converse does not hold, by the next example.

Given a ring $R$ and an $(R, R)$-bimodule $M$, the trivial extension of $R$ by $M$ is the ring $T(R, M)=R \oplus M$ with the usual addition and the following multiplication:

$$
\left(a_{1}, m_{1}\right)\left(a_{2}, m_{2}\right)=\left(a_{1} a_{2}, a_{1} m_{2}+m_{1} a_{2}\right) .
$$

This is isomorphic to the ring of all matrices $\left(\begin{array}{cc}a & m \\ 0 & a\end{array}\right)$, where $a \in R$ and $m \in M$ and the usual matrix operations are used.

Example 3.4 [14, Example 2]. Let $D$ be a domain and let $R=T(D, D)$ be the trivial extension of $D$. Then $R$ has the IFP and $R$ is a generalized right $P P$-ring, but it is not a right $P P$-ring. Thus $R$ is a generalized right p.q.-Baer ring by Proposition 3.3, but it is not right p.q.-Baer by [8, Proposition 1.14].

Recall from [5] that an idempotent $e \in R$ is called left (resp., right) semicentral if $x e=$ exe (resp., ex $=$ exe) for all $x \in R$. The set of left (resp., right) semicentral idempotents of $R$ is denoted by $S_{\ell}(R)$ (resp., $S_{r}(R)$ ). Note that $S_{\ell}(R) \cap S_{r}(R)=\mathbf{B}(R)$, where $\mathbf{B}(R)$ is the set of all central idempotents of $R$, and if $R$ is semiprime then $S_{\ell}(R)=S_{r}(R)=\mathbf{B}(R)$. Some of the basic properties of these idempotents are indicated in the following.

Lemma 3.5 [7, Lemma 1.1]. For an idempotent $e \in R$, the following are equivalent:

(1) $e \in S_{\ell}(R)$;

(2) $1-e \in S_{r}(R)$;

(3) $(1-e) R e=0$;

(4) $e R$ is a two-sided ideal of $R$;

(5) $R(1-e)$ is a two-sided ideal of $R$.

The following example shows that the condition " $R$ has the IFP" in Proposition 3.3 cannot be dropped. 
Example 3.6 [8, Example 1.6]. For a field $F$, take $F_{n}=F$ for $n=1,2, \ldots$, and let

$$
R=\left(\begin{array}{lc}
\prod_{n=1}^{\infty} F_{n} & \bigoplus_{n=1}^{\infty} F_{n} \\
\bigoplus_{n=1}^{\infty} F_{n} & \left\langle\bigoplus_{n=1}^{\infty} F_{n}, 1\right\rangle
\end{array}\right),
$$

which is a subring of the $2 \times 2$ matrix ring over the ring $\prod_{n=1}^{\infty} F_{n}$, where $<\bigoplus_{n=1}^{\infty} F_{n}, 1>$ is the $F$-algebra generated by $\oplus_{n=1}^{\infty} F_{n}$ and 1 . Then $R$ is a regular ring by [10, Lemma 1.6], and so $R$ is a generalized $P P$-ring.

Let $a \in\left(a_{n}\right) \in \prod_{n=1}^{\infty} F_{n}$ such that $a_{n}=1$ if $n$ is odd and $a_{n}=0$ if $n$ is even, and let $\alpha=$ $\left(\begin{array}{ll}a & 0 \\ 0 & 0\end{array}\right) \in R$. Now we assume that there exists an idempotent $e \in R$ such that $r_{R}\left(\alpha^{k} R\right)=e R$ for a positive integer $k$. Then $e$ is left semicentral, and so $e$ is central since $R$ is semiprime, but this is impossible. Thus $R$ is not generalized right p.q.-Baer. Similarly $R$ is not generalized left p.q.-Baer.

Proposition 3.7. Let $R$ be a ring. The following are equivalent:

(1) $R$ is generalized right p.q.-Baer;

(2) for any principal ideal I of the form $R a^{n} R$ of $R$, where $n$ is a positive integer, there exists $e \in S_{r}(R)$ such that $I \subseteq R e$ and $r_{R}(I) \cap R e=(1-e) R e$.

Proof. The proof is an adaptation from [8, Proposition 1.9]. (1) $\Rightarrow(2)$. Assume (1) holds. Then $r_{R}(I)=r_{R}\left(R a^{n} R\right)=r_{R}\left(a^{n} R\right)=f R$ with $f \in S_{\ell}(R)$. So $I \subseteq \ell_{R}\left(r_{R}(I)\right)=R(1-f)$. Let $e=1-f$, then $e \in S_{r}(R)$. Hence $r_{R}(I) \cap R e=(1-e) R \cap R e=(1-e) R e$.

$(2) \Rightarrow(1)$. Assume (2) holds. Clearly $(1-e) R \subseteq r_{R}(I)$ for any ideal $I$ of the form $R a^{n} R$. Let $\alpha \in r_{R}(I)$, then $\alpha e=e \alpha e+(1-e) \alpha e \in r_{R}(I) \cap R e=(1-e) R e$. So $e \alpha=e \alpha e=0$. Hence $\alpha=(1-e) \alpha \in(1-e) R$. Thus $r_{R}(I)=(1-e) R$, and therefore $R$ is generalized right p.q.Baer.

COROLlaRY 3.8. Let $R$ be a generalized right p.q.-Baer ring. If $I$ is a principal ideal of the form $R a^{n} R$ of $R$, then there exists $e \in S_{r}(R)$ such that $I \subseteq R e,(1-e) R e$ is an ideal of $R$, and $I+(1-e) R e$ is left essential in Re.

As a parallel result to [8, Proposition 1.12], we have the following whose proof is also an adaptation from [8].

Proposition 3.9. If $R$ is a generalized right p.q.-Baer ring, then the center $C(R)$ of $R$ is a generalized PP-ring.

Proof. Let $a \in C(R)$. For any positive integer $n$, there exists $e \in S_{\ell}(R)$ such that $\ell_{R}\left(a^{n}\right)=$ $\ell_{R}\left(R a^{n}\right)=r_{R}\left(a^{n}\right)=r_{R}\left(a^{n} R\right)=e R$. Observe that $\ell_{R}\left(R a^{n}\right)=\ell_{R} r_{R} \ell_{R}\left(R a^{n}\right)=\ell_{R} r_{R}(e R)$. Let $r_{R}(e R)=r_{R}\left(e^{n} R\right)=f R$ with $f \in S_{\ell}(R)$, then $1-f \in S_{r}(R)$. Hence $e R=\ell_{R}\left(R a^{n}\right)=$ $\ell_{R} r_{R}(e R)=\ell_{R}(f R)=R(1-f)$. So there exists $x \in R$ such that $e=x(1-f)$, and hence $e f=x(1-f) f=0$. Now $f e=e f e=0$ because $e \in S_{\ell}(R)$, and so $e f=f e=0$. Since $e R=R(1-f)$, there is $y \in R$ such that $1-f=e y$, and so $e=e(1-f)=e y=1-f$. Thus $e \in S_{\ell}(R) \cap S_{r}(R)=\mathbf{B}(R)$. Consequently, $r_{C}(R)\left(a^{n}\right)=r_{R}\left(a^{n}\right) \cap C(R)=e R \cap C(R)=$ $e C(R)$. Therefore the center $C(R)$ of $R$ is a generalized $P P$-ring. 
The following example shows that there exists a semiprime ring $\mathscr{R}$ whose center is a generalized $P P$, but $\mathscr{R}$ is not a generalized right p.q.-Baer.

Example 3.10. Let $\mathscr{R}=R \oplus \mathrm{Mat}_{2}(\mathbb{Z}[x])$, where

$$
R=\left(\begin{array}{lc}
\prod_{n=1}^{\infty} F_{n} & \bigoplus_{n=1}^{\infty} F_{n} \\
\bigoplus_{n=1}^{\infty} F_{n} & \left\langle\bigoplus_{n=1}^{\infty} F_{n}, 1\right\rangle
\end{array}\right)
$$

in Example 3.6. Then the center of $\mathscr{R}$ is generalized $P P$. Since $R$ is not a generalized right p.q.-Baer by Example 3.6, $\mathscr{R}$ is not a generalized right p.q.-Baer either. Furthermore, due to [14, Example 4], $\mathrm{Mat}_{2}(\mathbb{Z}[x])$ is not a generalized right $P P$. Thus $\mathscr{R}$ is not generalized right $P P$.

Note that given a reduced ring $R$, the trivial extension of $R$ (by $R$ ) has the IFP by simple computations. However, the trivial extension of a ring $R$ which has the IFP does not have the IFP by [13, Example 11]. We give examples of generalized right p.q.-Baer rings, which are extensions of the trivial extension, as in the following.

LEMMA 3.11. Let $S$ be a ring and for $n \geq 2$,

$$
R_{n}=\left\{\left(\begin{array}{ccccc}
a & a_{12} & a_{13} & \cdots & a_{1 n} \\
0 & a & a_{23} & \cdots & a_{2 n} \\
0 & 0 & a & \cdots & a_{3 n} \\
\vdots & \vdots & \vdots & \ddots & \vdots \\
0 & 0 & 0 & \cdots & a
\end{array}\right) \mid a, a_{i j} \in S\right\}
$$

If $S$ has the IFP, then for any $A \in R_{n}$ and any $E^{2}=E \in R_{n}, A E=\mathbf{0}$ implies that $A R_{n} E=\mathbf{0}$, where $\mathbf{0}$ is the zero matrix in $R_{n}$.

Proof. Note that every idempotent $E$ in $R_{n}$ is of the form

$$
\left(\begin{array}{ccccc}
e & 0 & 0 & \cdots & 0 \\
0 & e & 0 & \cdots & 0 \\
0 & 0 & e & \cdots & 0 \\
\vdots & \vdots & \vdots & \ddots & \vdots \\
0 & 0 & 0 & \cdots & e
\end{array}\right)
$$

with $e^{2}=e \in S$ by [14, Lemma 2]. Suppose that $A E=\mathbf{0}$ for any

$$
A=\left(\begin{array}{ccccc}
a & a_{12} & a_{13} & \cdots & a_{1 n} \\
0 & a & a_{23} & \cdots & a_{2 n} \\
0 & 0 & a & \cdots & a_{3 n} \\
\vdots & \vdots & \vdots & \ddots & \vdots \\
0 & 0 & 0 & \cdots & a
\end{array}\right) \in R_{n}
$$


Then we have the following: $a e=0$ and $a_{i j} e=0$ for $i<j, 1 \leq i$ and $2 \leq j$. Since $S$ has the IFP, $a S e=0$ and $a_{i j} S e=0$ for $i<j, 1 \leq i$ and $2 \leq j$. These imply that $A R_{n} E=\mathbf{0}$.

Proposition 3.12. Let a ring S have the IFP and let $R_{n}$ for $n \geq 2$ be the ring in Lemma 3.11. Then the following are equivalent:

(1) $S$ is generalized right p.q.-Baer;

(2) $R_{n}$ is generalized right $P P$;

(2) $R_{n}$ is generalized right p.q.-Baer.

Proof. $(1) \Rightarrow(2)$. Suppose that $S$ is generalized right p.q.-Baer. By Proposition 3.3, $S$ is a generalized right $P P$. Hence $R_{n}$ is also a generalized right $P P$ by [14, Proposition 3].

$(2) \Rightarrow(3)$. Suppose that $R_{n}$ is a generalized right $P P$. Then for any

$$
A=\left(\begin{array}{ccccc}
a & a_{12} & a_{13} & \cdots & a_{1 n} \\
0 & a & a_{23} & \cdots & a_{2 n} \\
0 & 0 & a & \cdots & a_{3 n} \\
\vdots & \vdots & \vdots & \ddots & \vdots \\
0 & 0 & 0 & \cdots & a
\end{array}\right) \in R_{n}
$$

and a positive integer $k$, there exists an idempotent

$$
E=\left(\begin{array}{ccccc}
e & 0 & 0 & \cdots & 0 \\
0 & e & 0 & \cdots & 0 \\
0 & 0 & e & \cdots & 0 \\
\vdots & \vdots & \vdots & \ddots & \vdots \\
0 & 0 & 0 & \cdots & e
\end{array}\right) \in R_{n}
$$

with $e^{2}=e \in S$ such that $r_{R_{n}}\left(A^{k}\right)=E R_{n}$. Note that $r_{R_{n}}\left(A^{k} R_{n}\right) \subseteq E R_{n}$. From $r_{R_{n}}\left(A^{k}\right)=$ $E R_{n}, A^{k} E=\mathbf{0}$, and so $A^{k} R_{n} E=\mathbf{0}$ by Lemma 3.11. Thus we have $E \in r_{R_{n}}\left(A^{k} R_{n}\right)$, and so $E R_{n} \subseteq r_{R_{n}}\left(A^{k} R_{n}\right)$. Consequently, $r_{R_{n}}\left(A^{k} R_{n}\right)=E R_{n}$, and therefore $R_{n}$ is generalized right p.q.-Baer.

(3) $\Rightarrow(1)$. Suppose that $R_{n}$ is a generalized right p.q.-Baer. Let $a \in S$ and consider

$$
A=\left(\begin{array}{ccccc}
a & 0 & 0 & \cdots & 0 \\
0 & a & 0 & \cdots & 0 \\
0 & 0 & a & \cdots & 0 \\
\vdots & \vdots & \vdots & \ddots & \vdots \\
0 & 0 & 0 & \cdots & a
\end{array}\right) \in R_{n}
$$


Since $R_{n}$ is a generalized right p.q.-Baer, $r_{R_{n}}\left(A^{k} R_{n}\right)=E R_{n}$ for some $E^{2}=E \in R_{n}$ and a positive integer $k$. Then by [14, Lemma 2], there is $e^{2}=e \in S$ such that

$$
E=\left(\begin{array}{ccccc}
e & 0 & 0 & \cdots & 0 \\
0 & e & 0 & \cdots & 0 \\
0 & 0 & e & \cdots & 0 \\
\vdots & \vdots & \vdots & \ddots & \vdots \\
0 & 0 & 0 & \cdots & e
\end{array}\right) \in R_{n}
$$

Hence $e S \subseteq r_{S}\left(a^{k} S\right)$. Let $b \in r_{S}\left(a^{k} S\right)$, then

$$
\left(\begin{array}{ccccc}
b & 0 & 0 & \cdots & 0 \\
0 & b & 0 & \cdots & 0 \\
0 & 0 & b & \cdots & 0 \\
\vdots & \vdots & \vdots & \ddots & \vdots \\
0 & 0 & 0 & \cdots & b
\end{array}\right) \in R_{n}
$$

is contained in $r_{R_{n}}\left(A^{k} R_{n}\right)=E R_{n}$, so $b \in e S$. Thus $S$ is also a generalized right p.q.-Baer ring.

\section{Acknowledgments}

The author would like to thank the referee for his/her helpful comments which improved this paper. This work was supported by the Daejin University Research Grants in 2004.

\section{References}

[1] E. P. Armendariz, A note on extensions of Baer and P.P.-rings, Journal of the Australian Mathematical Society, Series A 18 (1974), 470-473.

[2] H. E. Bell, Near-rings in which each element is a power of itself, Bulletin of the Australian Mathematical Society 2 (1970), 363-368.

[3] S. K. Berberian, Baer *-Rings, Die Grundlehren der mathematischen Wissenschaften, Band 195, Springer, New York, 1972.

[4] G. F. Birkenmeier, Baer rings and quasicontinuous rings have a MDSN, Pacific Journal of Mathematics 97 (1981), no. 2, 283-292.

[5] , Idempotents and completely semiprime ideals, Communications in Algebra 11 (1983), no. $6,567-580$.

[6] ___ Decompositions of Baer-like rings, Acta Mathematica Hungarica 59 (1992), no. 3-4, 319326.

[7] G. F. Birkenmeier, J. Y. Kim, and J. K. Park, Quasi-Baer ring extensions and biregular rings, Bulletin of the Australian Mathematical Society 61 (2000), no. 1, 39-52.

[8] , Principally quasi-Baer rings, Communications in Algebra 29 (2001), no. 2, 639-660.

[9] W. E. Clark, Twisted matrix units semigroup algebras, Duke Mathematical Journal 34 (1967), no. 3, 417-423.

[10] K. R. Goodearl, Von Neumann Regular Rings, Monographs and Studies in Mathematics, vol. 4, Pitman, Massachusetts, 1979.

[11] Y. Hirano, On generalized p.p.-rings, Mathematical Journal of Okayama University 25 (1983), no. 1, 7-11. 
[12] C. Y. Hong, N. K. Kim, and T. K. Kwak, Ore extensions of Baer and p.p.-rings, Journal of Pure and Applied Algebra 151 (2000), no. 3, 215-226.

[13] _ Extensions of generalized reduced rings, Algebra Colloquium 12 (2005), no. 2, 229-240.

[14] C. Huh, H. K. Kim, and Y. Lee, p.p. rings and generalized p.p. rings, Journal of Pure and Applied Algebra 167 (2002), no. 1, 37-52.

[15] I. Kaplansky, Rings of Operators, Math. Lecture Note Series, Benjamin, New York, 1965.

[16] M. Ôhori, On noncommutative generalized p.p. rings, Mathematical Journal of Okayama University 26 (1984), 157-167.

[17] A. Pollingher and A. Zaks, On Baer and quasi-Baer rings, Duke Mathematical Journal 37 (1970), $127-138$.

[18] L. H. Rowen, Ring Theory. Vol. II, Pure and Applied Mathematics, vol. 128, Academic Press, Massachusetts, 1988.

[19] G. Shin, Prime ideals and sheaf representation of a pseudo symmetric ring, Transactions of the American Mathematical Society 184 (1973), 43-60 (1974).

Tai Keun Kwak: Department of Mathematics, Daejin University, Pocheon 487-711, South Korea

E-mail address: tkkwak@daejin.ac.kr 


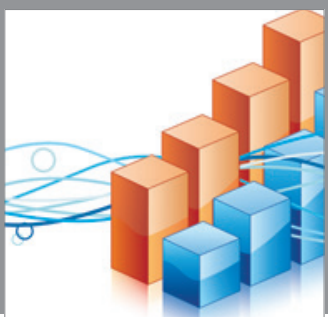

Advances in

Operations Research

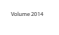

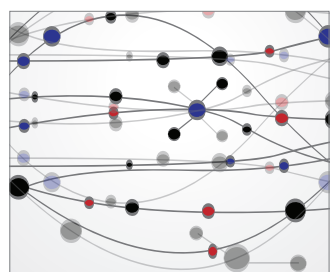

\section{The Scientific} World Journal
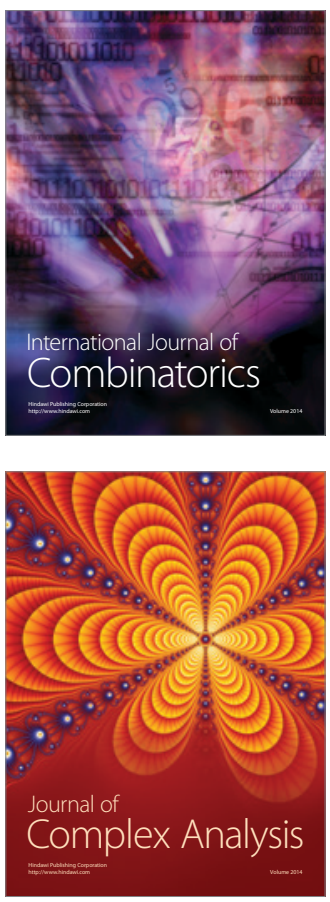

International Journal of

Mathematics and

Mathematical

Sciences
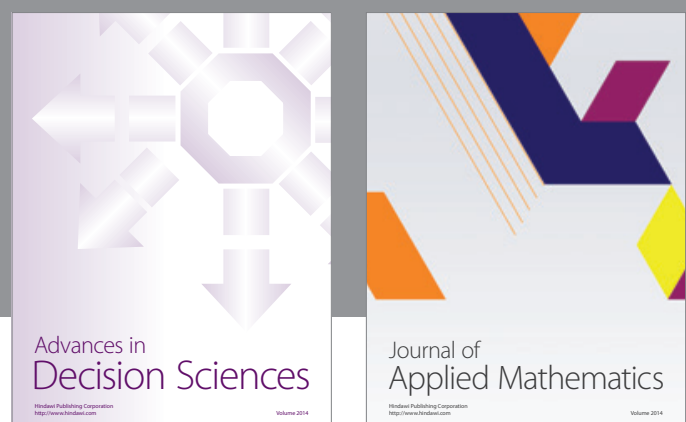

Journal of

Applied Mathematics
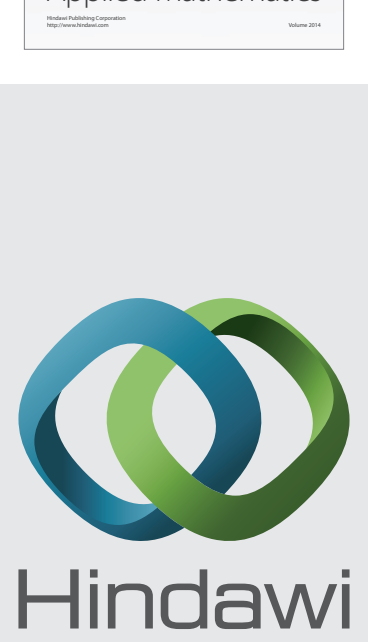

Submit your manuscripts at http://www.hindawi.com
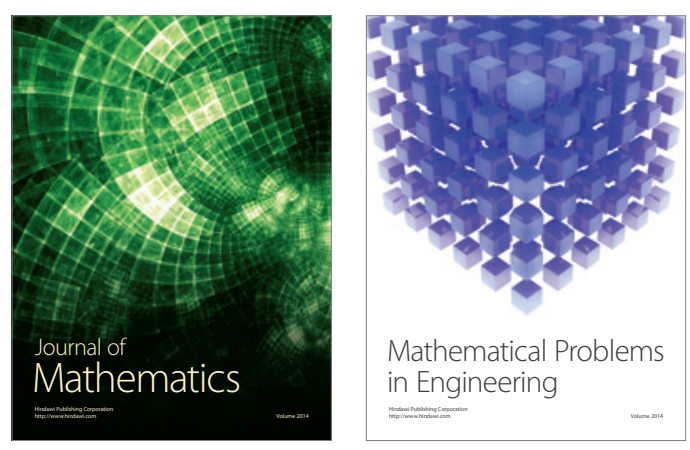

Mathematical Problems in Engineering
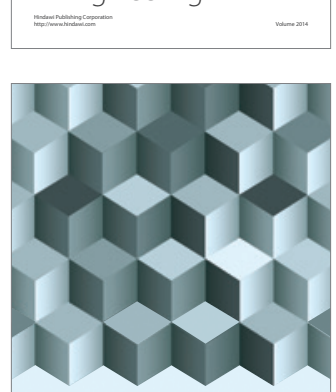

Journal of

Function Spaces
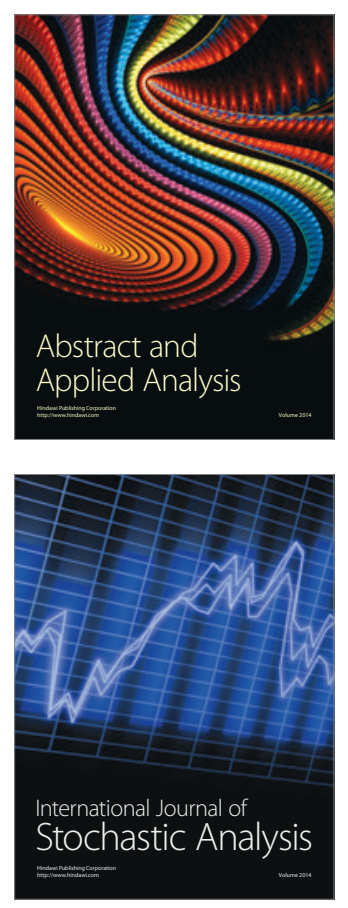

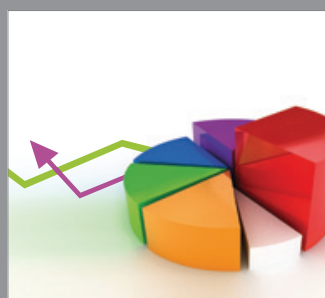

ournal of

Probability and Statistics

Promensencen
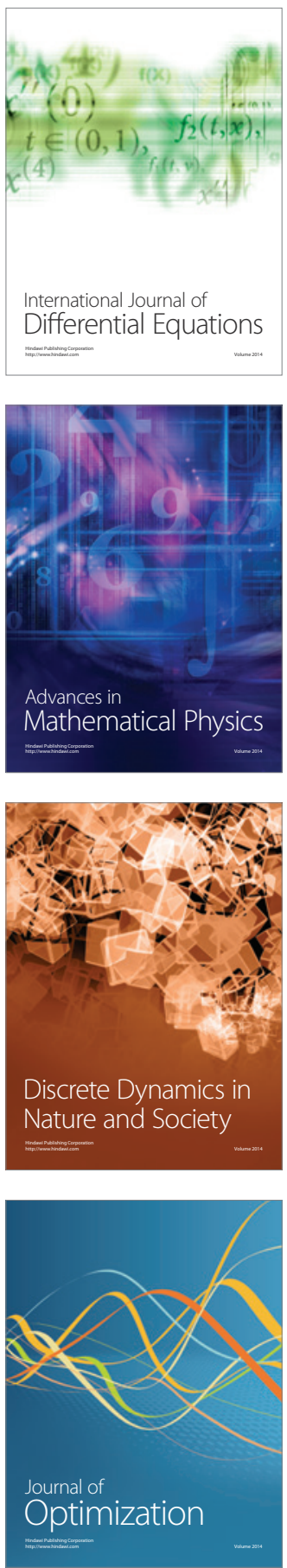